\title{
Proof of a conjecture of Zahariuta concerning a problem of Kolmogorov on the $\epsilon$-entropy
}

\section{Stéphanie Nivoche}

Laboratoire de Mathématiques Emile Picard, C.N.R.S.-U.M.R. 5580, Université Paul SabatierU.F.R. M.I.G., 118, route de Narbonne, 31062 Toulouse-Cedex, France

(e-mail: nivoche@picard.ups-tlse.fr)

Invent. math., http://dx.doi.org/10.1007/s00222-004-0372-5

Published online: 2 September 2004

Due to an error, the wrong year of submission for the above paper was printed. Here are the correct dates:

Oblatum 9-V-2001 \& 22-III-2004 